\title{
Unraveling the multiscale damping properties of two-dimensional layered MXene
}

\section{Archana Loganathan MS}

Doctoral student, Nanomechanics and Nanotribology Laboratory, Department of Mechanical and Materials Engineering, Florida International University, Miami, FL, USA

\section{Pranjal Nautiyal BTech}

Doctoral student, Nanomechanics and Nanotribology Laboratory, Department of Mechanical and Materials Engineering, Florida International University, Miami, FL, USA
Benjamin Boesl PhD

Associate Professor, Nanomechanics and Nanotribology Laboratory, Department of Mechanical and Materials Engineering, Florida International University, Miami, FL, USA

Arvind Agarwal PhD

Professor and Chair, Nanomechanics and Nanotribology Laboratory, Department of Mechanical and Materials Engineering, Florida International University, Miami, FL, USA (corresponding author: agarwala@fiu.edu)

MXene has emerged as an exciting two-dimensional nanomaterial because of its interesting multifunctional properties. In this paper, the authors report the multiscale damping behaviors of pure MAX and MXene. Dynamic loading of a multilayer MXene assembly shows an appreciable loss tangent (tan $\delta$ ), indicating the energy-dissipation ability of the material. The $\tan \delta$ value of MXene is recorded to be as high as 0.37 , which is about a $200 \%$ improvement over that of pure MAX. It is hypothesized that there are multiscale energy-loss mechanisms active in the material. While intralayer bond contraction operates in individual MXene sheets, interlayer compression and sliding/shearing mechanisms are active between the stacked layers. The presence of functional groups, van der Waals interactions and a low coefficient of friction between the MXene sheets provide MXene with an extraordinary energy-dissipation ability. Damping behavior is highly stable in MXene for as high as 50000 cycles, making it extremely promising for advanced applications requiring superior impact resistance, stability against noise and ability to damp mechanical vibrations.

\section{Notation}

A Hamaker constant

E energy

$E^{\prime} \quad$ storage modulus

$E^{\prime \prime} \quad$ loss modulus

$E_{\mathrm{I}} \quad$ energy required for intralayer bond contracting within MXene sheet

$E_{\mathrm{II}} \quad$ energy required for interlayer compression between MXene sheets

$E_{\mathrm{III}} \quad$ energy required for interlayer shearing/sliding of MXene sheets

$F \quad$ force

$k_{\mathrm{S}} \quad$ spring constant

$R \quad$ radius of the spheres

$r \quad$ distance between two atoms

$S_{\mathrm{c}} \quad$ shear strength

$\tan \delta$ loss tangent

$x_{\mathrm{o}} \quad$ natural bond length between two atoms

$y_{\mathrm{o}} \quad$ natural bond length between an atom and a functional group

$\beta \quad$ damping constant

$\gamma \quad$ surface energy

$\delta \quad$ phase lag

$\varepsilon \quad$ coefficient of dissipation

$\tau \quad$ relaxation time

\section{Introduction}

The emergence of two-dimensional (2D) nanomaterials in the past decade is paving the way for the development of advanced materials with intriguing functionalities. ${ }^{1-4}$ Due to their 2D morphology, these materials are characterized by extraordinary surface areas and energies and display excellent mechanical, electrical, thermal, electrochemical, electromagnetic and catalytic properties. $^{5-9}$ Since the discovery of MXene in 2011, it has emerged as a unique 2D material. MXenes are obtained by exfoliation of layered ternary carbides or nitrides, also known as the MAX phase, which is chemically represented as $\mathrm{M}_{n+1} \mathrm{AX}_{n}$, where $\mathrm{M}$ is the transition metal, $\mathrm{A}$ is an $\mathrm{A}$ group element and $\mathrm{X}$ could be either nitrogen or carbon, with $n$ being 1,2 or $3 .^{10-12}$ The crystal structure of titanium-aluminum carbide $\left(\mathrm{Ti}_{2} \mathrm{AlC}\right)$, a typical MAX phase, is illustrated in Figure 1. It is a combination of ceramic titanium carbide $\left(\mathrm{Ti}_{2} \mathrm{C}\right)$ layers with metallic aluminum (Al) layers, where $\mathrm{Ti}-\mathrm{C}$ is a strong covalent bond, whereas $\mathrm{Ti}-\mathrm{Al}$ is a metallic bond and is relatively weaker. However, aluminum in the MAX phase is susceptible to chemical attack and reaction with oxygen in the atmosphere, degrading its properties and limiting its applications. ${ }^{10,13}$ Chemical etching of the metallic aluminum layer by hydrofluoric acid (HF) is a useful strategy to eliminate reactive aluminum from the MAX phase. ${ }^{14,15}$ This results in the formation of multilayer titanium carbide, known as MXene (Figure 1). MXene is characterized by a very high surface area ${ }^{15}$ (titanium carbide $-23 \mathrm{~m}^{2} / \mathrm{g}$ ), making it receptive to surface functionalization by $-\mathrm{OH},-\mathrm{F}$ or $-\mathrm{O}$ groups and to engineering of its physical and chemical properties for multifarious applications.

Since their discovery, MXenes have been studied for wide-ranging applications, such as batteries, supercapacitors, metal purifiers, sensors, electromagnetic shielding and hydrogen storage. ${ }^{15-21}$ However, the mechanical properties of MXene have been rarely studied and reported in the literature. The first-principles theoretical approach has been used to predict the mechanical properties of pristine MXene. Elastic properties of pristine MXene were 


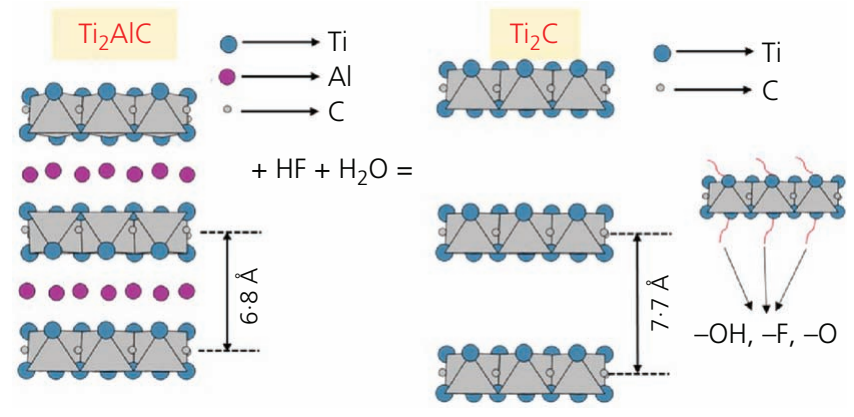

Figure 1. Schematic representation of the crystal structure of the MAX phase titanium-aluminum carbide $\left(\mathrm{Ti}_{2} \mathrm{AlC}\right)$ and the resulting MXene phase titanium carbide $\left(\mathrm{Ti}_{2} \mathrm{C}\right)$ after hydrofluoric acid treatment

obtained using first-principles density functional theory (DFT) and basal planes of MXene have been subjected to tensile and compressive deformations. This study revealed an impressive inplane elastic modulus, exceeding $600 \mathrm{GPa}^{22}$ Additionally, DFT calculations on structural, electronic, vibrational and mechanical properties of monolayer pristine and functionalized MXenes were performed. ${ }^{23-25}$ Wang et al..$^{23}$ revealed monolayer titanium carbide to be weaker than graphene but stronger than molybdenum disulfide $\left(\mathrm{MoS}_{2}\right)$. Guo et al. ${ }^{24}$ used first-principles calculations to demonstrate that titanium carbide functionalized with oxygen $(\mathrm{O})$ can withstand up to $28 \%$ strain due to its enhanced mechanical flexibility. This study showed $2 \mathrm{D} \quad \mathrm{Ti}_{n+1} \mathrm{C}_{n}$ as a potential candidate for flexible devices. Recently, Lipatov et al. ${ }^{26}$ measured the elastic properties of mono- and bi-layer $\mathrm{Ti}_{3} \mathrm{C}_{2} \mathrm{~T}_{x}$ ( $\mathrm{T}_{x}$ stands for surface termination) by indentation using atomic force microscopy. The bilayer $\mathrm{Ti}_{3} \mathrm{C}_{2} \mathrm{~T}_{x}$ expanded upon indentation, and the Young's modulus measured on monolayer $\mathrm{Ti}_{3} \mathrm{C}_{2} \mathrm{~T}_{x}$ was $\sim 0.33 \mathrm{TPa}^{26}$ The authors report this to be the highest modulus among other 'solution-processed' 2D materials. MXene is also found to exhibit pseudo-negative compressibility, as it undergoes expansion along its crystallographic $c$ direction when subjected to compression in the presence of water. This is accomplished by extensive slipping between the individual layers. ${ }^{27}$

Based on these studies on mechanical properties of MXene, researchers have attempted to use it as a promising reinforcement candidate for developing nanocomposites. ${ }^{28,29}$ Ling et al. ${ }^{28}$ demonstrated that the addition of $40 \mathrm{wt} . \% \mathrm{Ti}_{3} \mathrm{C}_{2} \mathrm{~T}_{x}$ to poly(vinyl alcohol) (PVA) matrix film by vacuum-assisted filtration resulted in a $200 \%$ increase in stiffness and tensile strength due to functionalization by the $-\mathrm{OH}$ group, which improved the interfacial bonding between the MXene sheets and PVA. The tribological properties of titanium carbide $\left(\mathrm{Ti}_{3} \mathrm{C}_{2}\right)$ MXene have drawn significant attention among researchers. ${ }^{30-37}$ Liu et al. ${ }^{32}$ studied $\mathrm{Ti}_{3} \mathrm{C}_{2}$ MXene as an additive to polyalphaolefin base oil. Lower coefficient of friction (COF) $(\sim 0 \cdot 11)$ and wear volume $(0 \cdot 5 \times$ $10^{4} \mu \mathrm{m}^{3}$ ) were obtained for $0 \cdot 8 \% \mathrm{Ti}_{3} \mathrm{C}_{2}$ MXene concentration, due to sliding friction and continuous tribofilm formation on the surface. Similarly, $\mathrm{Ti}_{3} \mathrm{C}_{2}$-reinforced ultra-high-molecular-weight polyethylene (UHMWPE) composite sheets were prepared through the hot-press method at $220^{\circ} \mathrm{C}$ and $10 \mathrm{MPa}$ pressure with $30 \mathrm{~min}$ of dwell time. $\mathrm{Ti}_{3} \mathrm{C}_{2} / \mathrm{UHMWPE}$ exhibited $31 \%$ a lower $\mathrm{COF}$ compared to pure UHMWPE because of the weak van der Waals force of interaction between $\mathrm{Ti}_{3} \mathrm{C}_{2}$ layers and improved crystallinity with the addition of $\mathrm{Ti}_{3} \mathrm{C}_{2}{ }^{35}$ MXene/copper $(\mathrm{Cu})$ composite coatings prepared through the electrodeposition technique used an aqueous solution of $\mathrm{Ti}_{3} \mathrm{C}_{2}$ and copper (II) disodium ethylenediaminetetraacetate tetrahydrate at a peak current density of $6 \mathrm{~A} / \mathrm{dm}^{2}$ and for $70 \mathrm{~min}$ duration. The MXene/copper composite coatings showed $\sim 46 \%$ reduced $\mathrm{COF}$ and $\sim 19 \%$ reduced wear rate compared to pure copper coatings. This is ascribed to easy shearing of $\mathrm{Ti}_{3} \mathrm{C}_{2}$ layers and dense tribofilm formation on the worn surface. ${ }^{37}$

Although there is a growing interest in MXene for enhancing the mechanotribological properties of composites, the current experimental understanding of fundamental deformation mechanisms of MXene is very limited. ${ }^{26,27}$ Due to their 2D morphology with a layered organization, MXenes are likely to exhibit a complex mechanical behavior with interplay between intralayer and interlayer deformation mechanisms. In addition to extraordinary intralayer strength and stiffness, interlayer van der Waals interactions between MXene layers are expected to give rise to a time-dependent mechanical response. This has been theoretically and experimentally demonstrated for another 2D material, graphene, where ripple formation and interlayer van der Waals interactions result in absorption and dissipation of mechanical energy. ${ }^{38,39}$ Twodimensional MXene sheets display morphology and structural organization analogous to that of graphene, suggesting the possibility of a damping behavior in MXene. However, the nature of intralayer bonding is different from that of graphene due to heteroatomic $\mathrm{Ti}-\mathrm{C}$ bonds as opposed to $\mathrm{sp}^{2}$-hybridized $\mathrm{C}-\mathrm{C}$ bonding in graphene. Therefore, unique deformation mechanisms are anticipated that are different from the deformation behavior of graphene. This study seeks to investigate these dynamic deformation characteristics of multilayer MXene.

In this study, the MAX phase was etched by hydrofluoric acid for varying time intervals (1,2 and $4 \mathrm{~h}$ ) to control the extent of aluminum etching and, in turn, tailor the nature of interlayer bonding. To probe the role of interlayer interactions on loadbearing characteristics, the damping properties of MAX and MXene are examined. Due to the hierarchical architecture of MXene, dynamic mechanical (DMA) tests were carried out at multiple load scales (from a few micronewtons to millinewtons) to activate intrinsic and interlayer deformation mechanisms selectively. This experimental study seeks to determine the correlation between the morphology, phase constitution, inter-/ intra-layer interactions and mechanics of pure MXene. Damping ability is crucial in mechanical structures and can open new avenues for advanced applications of MXene-based materials.

\section{Materials and methods}

\subsection{Synthesis}

Titanium-aluminum carbide MAX phase powder was obtained from Sandvik Materials Technology, Sweden. As-received 
titanium-aluminum carbide powder was immersed in $25 \%$ hydrofluoric acid solution for 1,2 and $4 \mathrm{~h}$ at room temperature to obtain the MXene phase. ${ }^{15,40}$ The solution was continuously stirred using a polytetrafluoroethylene (PTFE)-coated magnetic stirrer at 100 revolutions/min. After hydrofluoric acid treatment, powders were washed using deionized water and further separated by using the centrifugation technique. These powders were dried in a vacuum oven at $80^{\circ} \mathrm{C}$ for $30 \mathrm{~min}$ to $1 \mathrm{~h}$.

\subsection{Characterization}

Structural characterization of the as-received MAX and hydrofluoric acid-treated powders was carried out using an X-ray diffractometer (Siemens D5000) with copper $\mathrm{K} \alpha(\lambda=1 \cdot 542 \AA)$ radiation. A Jeol JSM-6330F field emission scanning electron microscope (SEM) was used to examine the microstructure of the as-received MAX powder and MXene. In order to perform the nanomechanical tests, the powder specimens were dispersed in ethanol by ultrasonication for $30 \mathrm{~min}$. The dispersed solution droplets were poured on a metallic stub, and ethanol was allowed to evaporate. This resulted in the deposition of MAX and hydrofluoric acid-treated particles on the stub. The tribological test was performed on the pure MAX and hydrofluoric acid-treated powders to elucidate the shearing/sliding of sheets; the experimental details of the test are given in the online supplementary material.

\subsection{Damping characterization}

The damping behaviors of pure MAX and hydrofluoric acidtreated specimens were examined at multiple load scales. NanoDMA tests were carried out on these deposited powder specimens using a TI 900 triboindenter (Hysitron Inc., Minneapolis, MN, USA). For nano-DMA, a dispersion of MAX/MXene particles in ethanol was produced by ultrasonication. This slurry was poured onto the stub. The particle concentration in ethanol was kept very low $(<5 \mathrm{mg} / \mathrm{ml})$ so as to obtain individual particles scattered around the stub (as shown in the optical micrograph of Figure S2 in the online supplementary material). Nano-DMA tests were performed on these 'individual' MXene particles. The indents were made on the top surface, as the powder particles were oriented due to their 2D morphology, and, prior to testing, this was confirmed by optically resolving the particles by using an optical microscope attached to the nanoindenter. The authors selectively probed the particles, as shown in Figures S1 and S2 in the online supplementary material. The indenter tip of the in situ nanoindenter (Bruker, Hysitron PI 87 SEM picoindenter) made contact with the top surface of MXene (Figure S1 in the online supplementary material). During the tests, cyclic dynamic loads of $10 \%$ were applied over a fixed static load using a Berkovich diamond tip of $100 \mathrm{~nm}$ diameter. The nano-DMA tests were conducted for low static loads of 10 and $100 \mu \mathrm{N}$, with corresponding dynamic loads of 1 and $10 \mu \mathrm{N}$, respectively. Damping characteristics are analyzed by determining the loss tangent $(\tan \delta$ ) over a range of frequencies from 10 to $250 \mathrm{~Hz}$. Also, the nanodynamic tests were conducted for 50000 cycles to examine the stability of the damping behavior over a large number of loading-unloading cycles. The indentation depths are less than
$10 \%$ of the particle thickness, as the particles are $\sim 3-5 \mu \mathrm{m}$ thick and $\sim 5-10 \mu \mathrm{m}$ wide. The indenter diameter is $\sim 100 \mathrm{~nm}$, which is more than an order of magnitude smaller than the particle size. Therefore, it is certain that the indentation loading is confined to a single particle. The average displacement/depth scale of these nanodynamic tests varies from about 9 to about $300 \mathrm{~nm}$.

The damping behavior at larger loads was studied by stylus impact tests conducted using an Innowep universal surface tester (Wurzburg, Germany) to obtain a dynamic response for a larger displacement range (up to $100 \mu \mathrm{m}$ ). For the high-load damping test, the particles were deposited onto the stub to obtain a coating/ film. This was done by making a slurry of MXene $(30 \mathrm{mg} / \mathrm{ml}$ powder-ethanol dispersion), pouring this slurry onto the stub and allowing the ethanol to evaporate. The powder concentration in ethanol was much higher compared to that of the nano-DMA samples. As a result, an MXene deposit was obtained, which can also be considered as a coating/film of $40-50 \mu \mathrm{m}$ thickness (Figure S3 in the online supplementary material). A spherical steel probe of $800 \mu \mathrm{m}$ diameter was used for these tests. The probe was dropped on the specimen, with applied loads of 1 and $5 \mathrm{mN}$, which are nearly three orders of magnitude greater than the loads applied in nano-DMA. The damped oscillations of the bouncing stylus generated the height-time profile and were recorded as raw data. An example of a height-time profile is shown in Figure 2(b). From the profile, the logarithmic decrement was obtained as the ratio of the heights of two successive extrema, and then the loss tangent $(\tan \delta$ ) was then computed from the logarithmic decrement $(\tan \delta=$ logarithmic decrement $/ \pi$ ). While the low-load nanodynamic tests using a fine nanosize probe capture the 'localized' damping behavior of a few layers of particles, the high-load-impact tests carried out using a larger probe measure the overall damping response from the aggregation of the particles. In addition, the samples were observed under an optical microscope before and after the damping tests, and there were no signs of loose particles, flaking or particle debonding due to stylus impact.

\section{Results and discussions}

\subsection{Structural and microstructural characterization}

X-ray diffraction (XRD) studies of the as-received MAX powder show the major phase to be titanium-aluminum carbide with a small amount of the titanium carbide (TiC) phase (Figure 3(a)). With hydrofluoric acid treatment of MAX powder, the intensity of the titanium-aluminum carbide phase decreases with broader peaks, indicating disorder in the crystal structure. Emergence of MXene phase was confirmed by shifting of the (002) peak at $12^{\circ}$ (titanium-aluminum carbide) to a smaller angle of $9^{\circ}$ along with peak broadening. This peak corresponds to titanium carbide formation (Figure 3(a)) with increasing ' $d$-spacing' $\left(d_{002}\right)$ or interlayer distance, from 6.75 to $8.45 \AA$. Also, the peak around $9^{\circ}$ was bifurcated for the 1 and $2 \mathrm{~h}$ hydrofluoric acid-treated samples, where both the peaks correspond to titanium carbide, indicating that MXene sheets have two different interlayer spacings. 


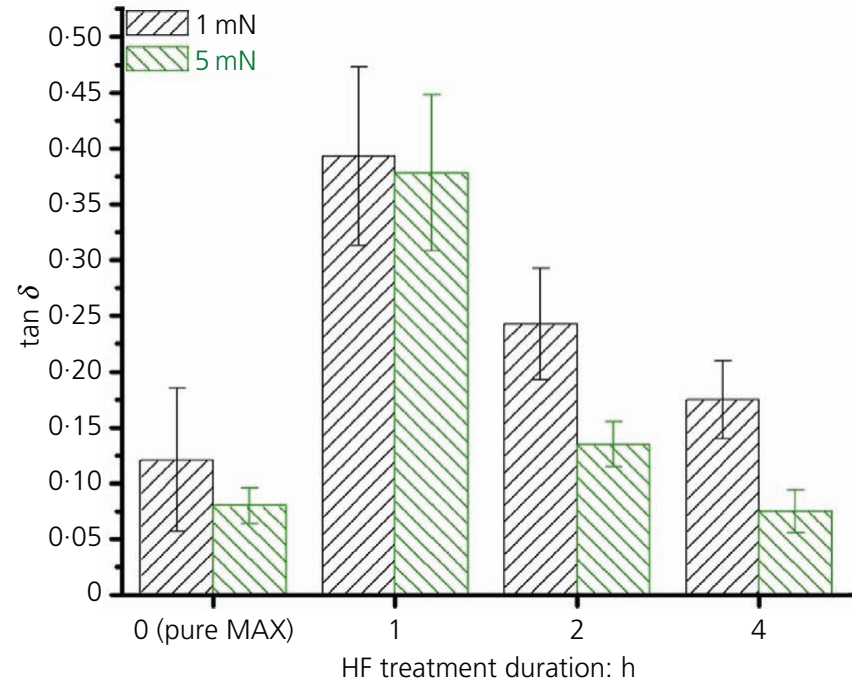

(a)

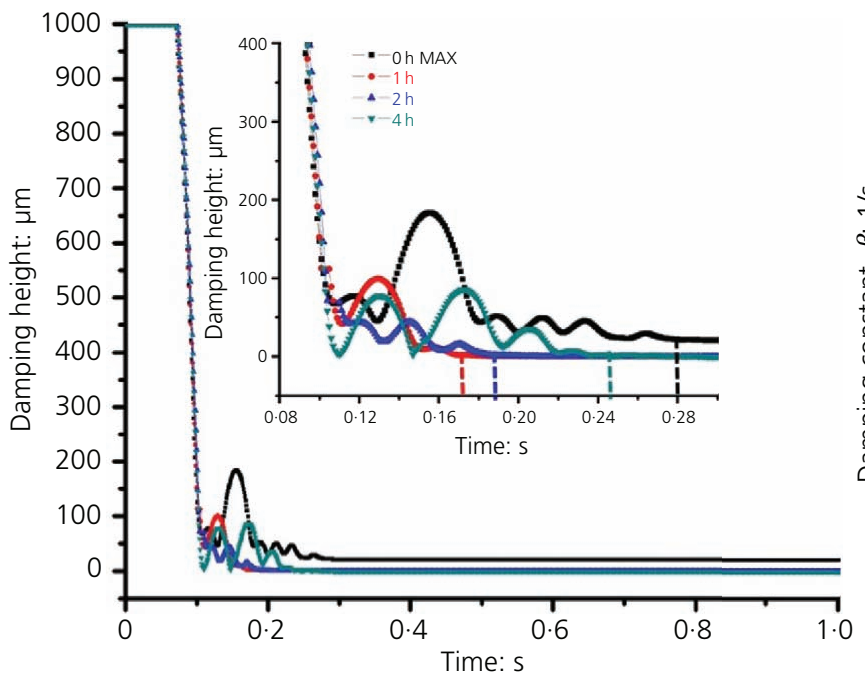

(c)

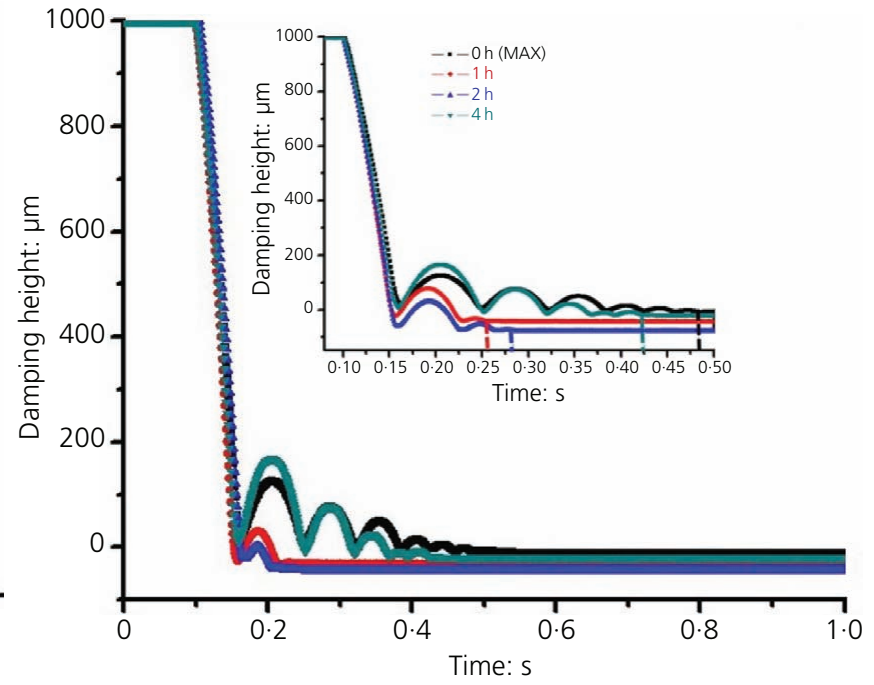

(b)

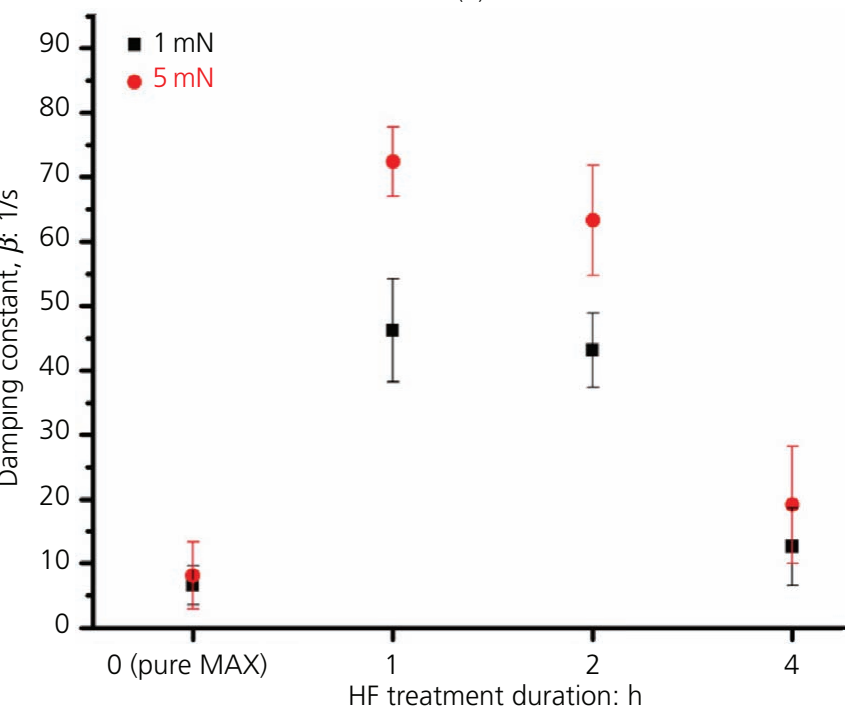

(d)

Figure 2. Comparison of (a) loss tangent values, (b) damping height-time profiles at $1 \mathrm{mN}$ load, (c) damping height-time profiles at $5 \mathrm{mN}$ load and (d) damping constant of pure MAX and hydrofluoric acid-treated samples. Damping behaviors of MAX and hydrofluoric acidtreated samples at dynamic loadings of (e) 1 and (f) $10 \mu \mathrm{N}$ in the frequency range 10-250 Hz. (g) Long cycle test for hydrofluoric acidtreated samples at loads of 1 and $10 \mu \mathrm{N}$ for a frequency of $150 \mathrm{~Hz}$ (continued on next page)

The interlayer spacing was calculated for all the hydrofluoric acid-treated MXene samples, and they are included in Table S1 in the online supplementary material. In the bifurcated (002) peaks, with increase in hydrofluoric acid treatment duration from 1 to $4 \mathrm{~h}$, the peak intensity at larger $d$-spacing remains constant with the other peak intensity almost weakening, indicating the increase in interlayer spacing with longer etching time. The new phase formation confirms the etching away of aluminum from the titanium-aluminum carbide structure. The resulting titanium carbide surfaces could possibly be terminated by $-\mathrm{OH},-\mathrm{F}$ and $-\mathrm{O}$ surface groups. The intensity of the MXene peaks decreases after $2 \mathrm{~h}$ of hydrofluoric acid treatment, whereas the intensity of titanium carbide peaks remains the same. In addition, broadening of the MXene peak was observed after $4 \mathrm{~h}$ of hydrofluoric acid treatment. This is ascribed to the slow decrease in the degree of structural order.

Figure 3(b) shows the SEM image of the as-received titaniumaluminum carbide powder. It shows a densely packed layered structure. After $1 \mathrm{~h}$ of hydrofluoric acid treatment, partial separation of the layers was observed (Figure 3(c)). The thickness of the separated layers was $157 \pm 20 \mathrm{~nm}$ for $1 \mathrm{~h}$ of etching. As the hydrofluoric acid treatment time was increased to $2 \mathrm{~h}$, further separation of the layers was observed, which is caused by the excess removal of aluminum from titanium-aluminum carbide, as shown in Figure 3(d). This leads to the formation of thin $(100 \pm 23 \mathrm{~nm})$ 


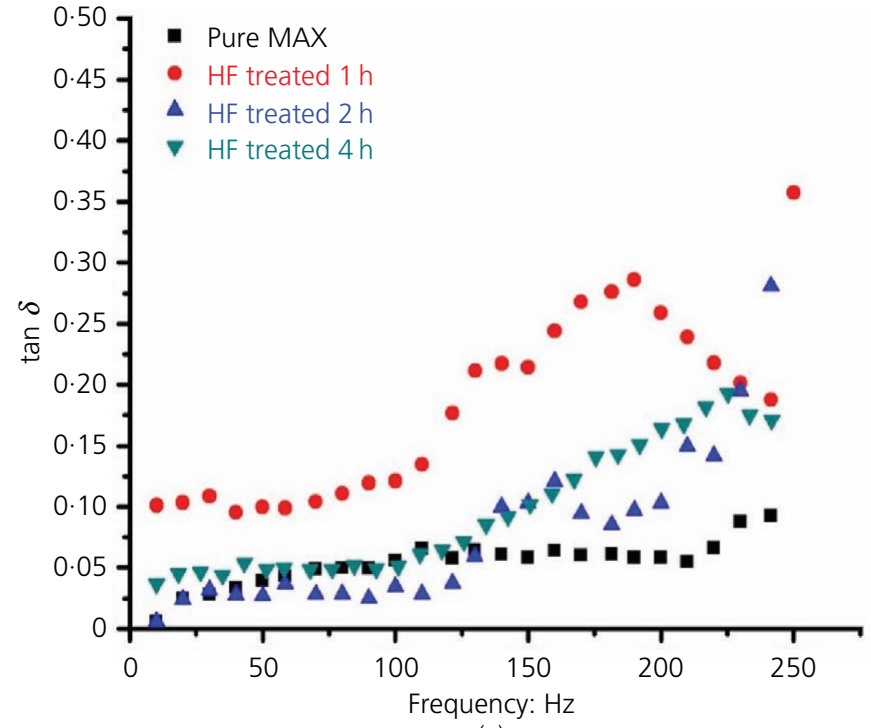

(e)

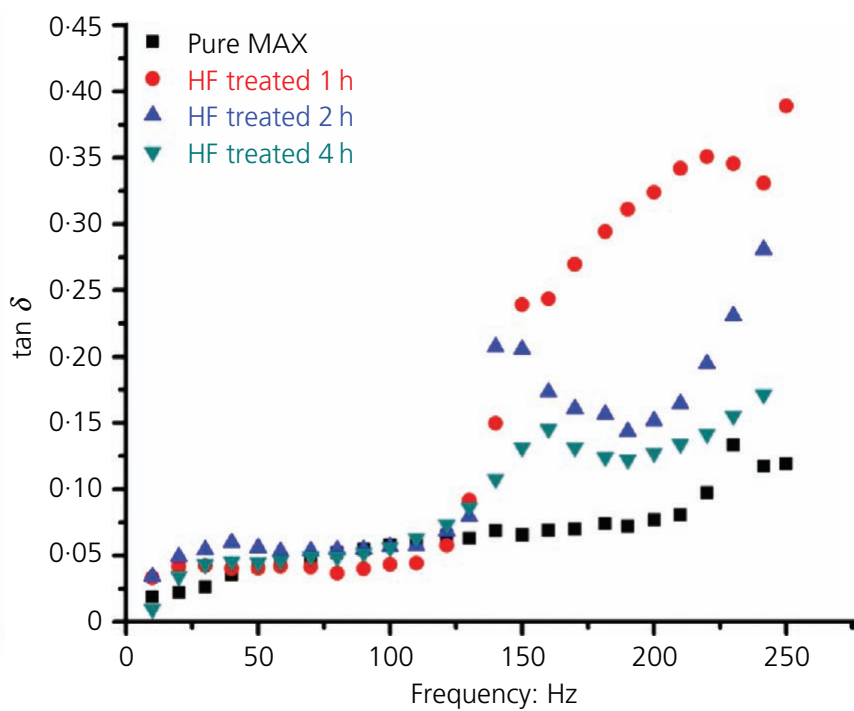

(f)

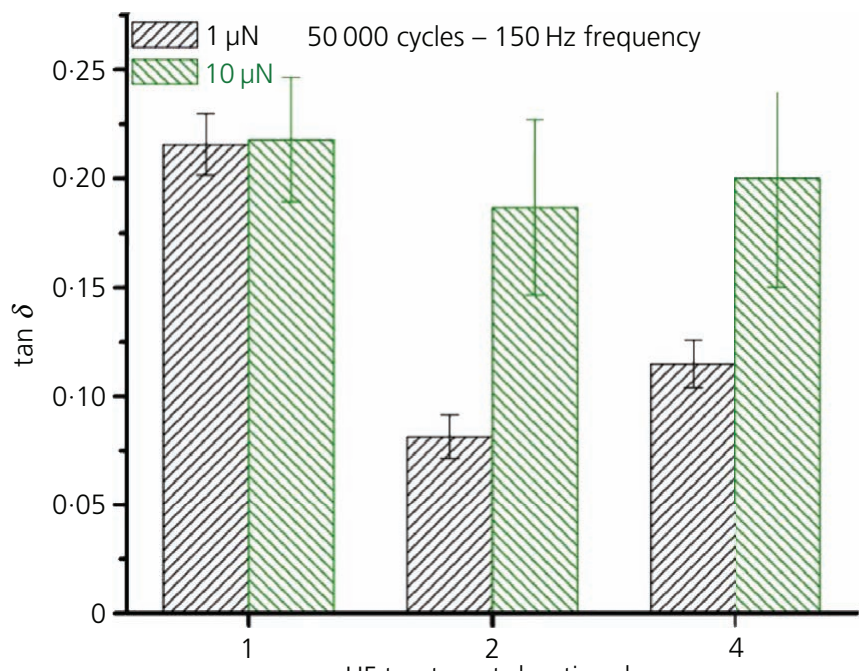

HF treatment duration: $h$

(g)

Figure 2. Continued

layers. Figure 3(e) reveals the hydrofluoric acid-treated $4 \mathrm{~h}$ powders to have evidently separated individual MXene layers with a reduced thickness of $74 \pm 12 \mathrm{~nm}$. The visual evidence shows a clean surface for all hydrofluoric acid-treated powders with no surface oxidation.

\subsection{Damping behavior of MAX and MXene}

For MAX and hydrofluoric acid-treated MXene samples, the damping behavior was examined at the multiscale level; the highload tests were performed at the millinewton scale and the lowload tests were performed at the micronewton scale (which is three orders of magnitude lower). The damping characteristics are analyzed by determining the phase lag $(\delta)$ between the applied force and displacement response. The loss tangent $(\tan \delta)$, which is a measure of damping capability, is given by the ratio of the loss modulus $\left(E^{\prime \prime}\right)$ to the storage modulus $\left(E^{\prime}\right)^{41}$
1. $\tan \delta=\frac{E^{\prime \prime}}{E^{\prime}}$

The loss modulus $\left(E^{\prime \prime}\right)$ represents the viscoelastic portion of the material, and the storage modulus $\left(E^{\prime}\right)$ represents the elastic part of the material. ${ }^{42}$ Figure 2(a) shows the $\tan \delta$ values measured at higher loads $(\mathrm{mN})$ applied along the top surface (normal to the basal plane) of all the powders. The loss tangent $(\tan \delta)$ value for the MAX phase fluctuates in the range $0 \cdot 05-0 \cdot 18$. After hydrofluoric acid treatment for $1 \mathrm{~h}$, the $\tan \delta$ value increases significantly from 0.32 to $0 \cdot 48$. An almost $200 \%$ increase in the loss tangent was observed compared to that of pure MAX, demonstrating an enhanced damping behavior, which could be directly correlated with the combination of damping mechanisms 


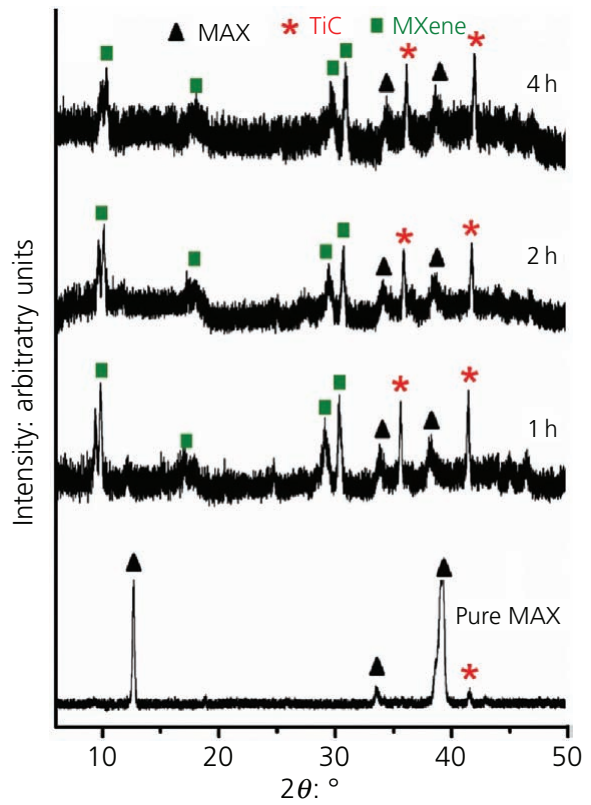

(a)

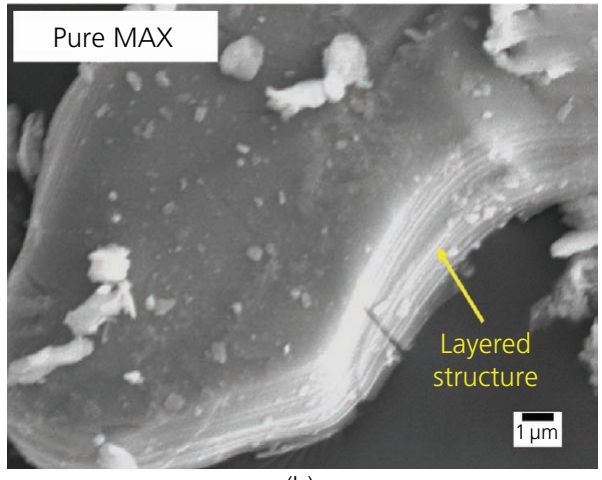

(b)

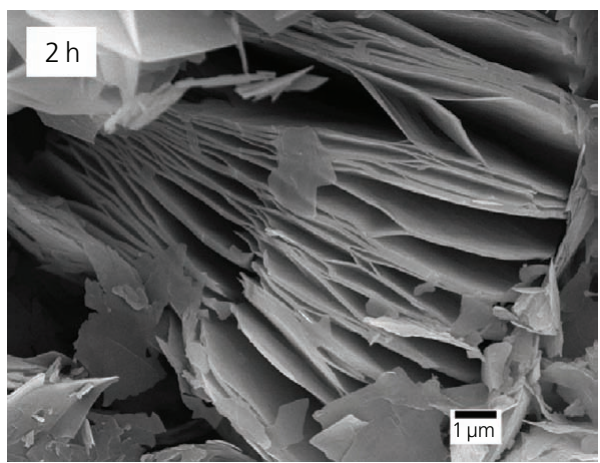

(d)

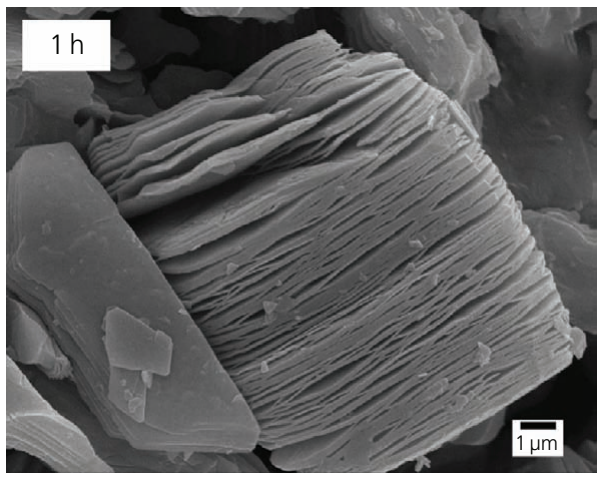

(c)

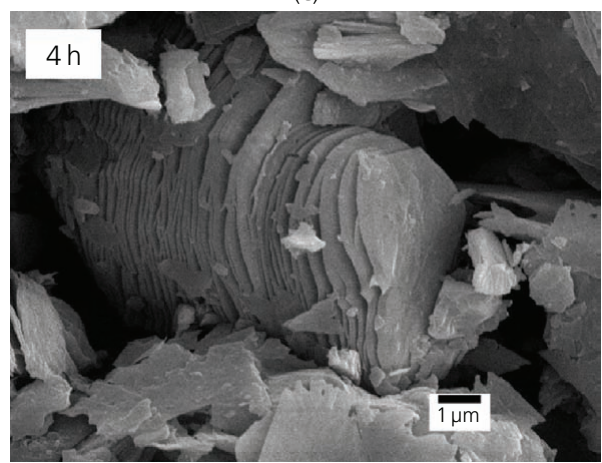

(e)

Figure 3. (a) XRD patterns of MAX and hydrofluoric acid-treated MXene powders; SEM images of (b) MAX, (c) $1 \mathrm{~h}$, (d) $2 \mathrm{~h}$ and (e) $4 \mathrm{~h}$ hydrofluoric acid-treated MXene powder

present in MAX and MXene phases. The dominant damping mechanisms for the MAX phase are the incipient kink band formation and ripplocations. ${ }^{43-47}$ The energy dissipation for MXene can be related to the intralayer bond contracting and interlayer mechanisms due to the weak van der Waals forces between the MXene sheets (discussed later in detail). Compared to pure MAX, the loss tangent remains higher for $2 \mathrm{~h}$ (about 98\%) and $4 \mathrm{~h}(40 \%)$ of hydrofluoric acid treatment. Among MXene samples, the loss tangent drops to 38 and $54 \%$ for the 2 and $4 \mathrm{~h}$ hydrofluoric acid-treated samples, respectively, compared to $1 \mathrm{~h}$ hydrofluoric acid treatment. It is noted in Figure 2(a) that, with increasing load from 1 to $5 \mathrm{mN}$, the loss tangent trends remain the 
same but the magnitude drops. This can be explained by the van der Waals forces between the 2D sheets of MXene. During dynamic loading, weak van der Waals interactions result in energy dissipation. However, as the applied loads become too high, the interlayer separation drops down significantly. At very low interlayer separation, the forces required to compress the sheets become too high. In such a scenario, the in-plane elastic deformation of the MXene sheets starts dominating over the viscous van der Waals interactions. Hence, the component of energy dissipation goes down, and this causes the drop in $\tan \delta$ with increasing load. The height-time profiles for both the loads ( 1 and $5 \mathrm{mN}$ ) of the damping test for MAX and hydrofluoric acidtreated samples are shown in Figures 2(b) and 2(c). This profile shows the period required for the amplitude of the oscillating stylus to decay through MAX and hydrofluoric acid-treated MXene. The pure MAX phase shows the longest decay times of $0.48 \mathrm{~s}(1 \mathrm{mN})$ and $0.28 \mathrm{~s}(5 \mathrm{mN})$ compared to hydrofluoric acidtreated MXene. The $1 \mathrm{~h}$ hydrofluoric acid-treated sample showed the maximum energy dissipation with the shortest decay times of $0 \cdot 25 \mathrm{~s}(1 \mathrm{mN})$ and $0 \cdot 17 \mathrm{~s}(5 \mathrm{mN})$ compared with $2 \mathrm{~h}(0 \cdot 28 \mathrm{~s}$ for $1 \mathrm{mN}$ and $0 \cdot 19 \mathrm{~s}$ for $5 \mathrm{mN})$ and $4 \mathrm{~h}(0.42 \mathrm{~s}$ for $1 \mathrm{mN}$ and $0.23 \mathrm{~s}$ for $5 \mathrm{mN}$ ) hydrofluoric acid-treated samples. This superior damping behavior of $1 \mathrm{~h}$ hydrofluoric acid-treated sample is substantiated by measuring the damping constant $(\beta$ ) (fitting the maxima and minima of the height-time profiles with exponential function) and is defined by the equation ${ }^{48}$

2. $\beta=\frac{1}{\tau}$

where $\tau$ is the relaxation time, described as the time span in which the amplitude of the oscillating stylus decays by a factor of 'e'. Figure 2(d) shows the damping constant $(\beta)$ of pure MAX and hydrofluoric acid-treated powders for both loads (1 and $5 \mathrm{mN}$ ). The MAX phase has the smallest damping constant compared to the hydrofluoric acid-treated samples, implying that the MAX phase undergoes slow damping with a longer relaxation time. The $1 \mathrm{~h}$ hydrofluoric acid-treated sample shows the highest damping constant, displaying rapid damping with a shorter relaxation time. The damping constant gradually decreases as the hydrofluoric acid treatment duration increases. This trend is similar to the $\tan \delta$ values and is attributed to the decrease in the titanium carbide layer thickness with longer etching period. Thus, the damping behavior changes with the decrease in the array of MXene layers.

A nanodynamic test was performed to probe the intrinsic damping behavior of a single or few layers of MXene, as opposed to the high-load test, which examines the overall damping behavior. Nano-DMA results for the samples at different loads ( 1 and $10 \mu \mathrm{N}$ ) are shown in Figures 2(e) and 2(f), where the $\tan \delta$ values $(0 \cdot 015-0 \cdot 10)$ change with respect to varying frequencies from 0 to $250 \mathrm{~Hz}$. The MAX phase displays a continuous increase in $\tan \delta$ values with an increasing frequency for both dynamic loads. Compared to MXene, the $\tan \delta$ increase for the MAX phase was gradual and plateaued out in the frequency range 100-200 Hz. Similar to high-load-damping tests, nano-DMA also shows higher $\tan \delta$ values for MXene varying from $0 \cdot 025$ to $0 \cdot 38$, indicating improved damping behavior compared with the pure MAX phase. Among the hydrofluoric acid-treated samples for $10 \mu \mathrm{N}$ (Figure 2(f)), it is observed that the $1 \mathrm{~h}$ treated sample has the highest loss tangent of $0 \cdot 38$, compared to the loss tangent of $2 \mathrm{~h}(0 \cdot 28)$ and $4 \mathrm{~h}$ $(0 \cdot 15)$ treated samples. With increasing frequency for both dynamic loading conditions, the hydrofluoric acid-treated samples exhibit an upsurge of $\tan \delta$, with a minimum value of 0.025 to a maximum value of $0 \cdot 38$. Particularly for hydrofluoric acid-treated MXene, there is a gradual increase in $\tan \delta$ up to $125 \mathrm{~Hz}$, and it jumps to a higher value at $150 \mathrm{~Hz}$. Beyond $200 \mathrm{~Hz}$, the 1 and $2 \mathrm{~h}$ hydrofluoric acid-treated samples display a gradually decreasing $\tan \delta$ up to $250 \mathrm{~Hz}$. This displays the significant role of frequency in the damping behavior. Regardless of high or low damping loads and frequency, higher $\tan \delta$ values for MXene indicate the synergistic contribution of intralayer and interlayer damping mechanisms from the MXene layers. The stability of the nanoscale damping response in MXene samples was studied by subjecting them to a large number of cycles (50000 cycles) at a frequency of $150 \mathrm{~Hz}$ for 1 and $10 \mu \mathrm{N}$ loads. For this study, the frequency of $150 \mathrm{~Hz}$ was chosen for both loads as a significant leap in the $\tan \delta$ value was observed during the nanodynamic tests (Figures 2(e) and 2(f)). Figure 2(g) shows that MXene retains a stable damping behavior. At $1 \mu \mathrm{N}$ load, $\tan \delta$ remained constant for the $1 \mathrm{~h}$ sample with a small standard deviation, indicating that the damping process has not degraded the 2D layers after 50000 cycles. For $10 \mu \mathrm{N}$ load, all of the three hydrofluoric acid-treated conditions $(1,2$ and 4 h) displayed similar $\tan \delta$ values with a higher standard deviation. In this long cycle test, an increase in $\tan \delta$ is observed with increasing load from 1 to $10 \mu \mathrm{N}$. At low load, the mechanism from a single sheet (intralayer bond contraction) is more prominent due to localized dynamic loading using a fine, Berkovich tip of $100 \mathrm{~nm}$ radius. Increasing load results in enhanced bond distortion. Thus, $\tan \delta$ is found to increase with load. This persistent damping behavior of hydrofluoric acid-treated samples after 50000 cycles clearly implies the efficient energy dissipation dictated by MXene upon cyclic loading.

\subsection{Damping mechanisms in MAX and MXene}

It is evident from the multiscale damping studies that the MXene phase displayed superior damping behavior compared to pure MAX for both low and higher loads. It is also concluded that the nanoscale damping behavior is largely stable up to 50000 cycles. Energy dissipation in MXene can be elucidated by three possible mechanisms, namely

(a) intralayer - bond contracting within an MXene sheet

(b) interlayer - compression between MXene sheets

(c) interlayer - sliding/shearing between the sheets.

(a) Intralayer - bond contracting: as a load is applied to an MXene sheet, the bond length of covalently bonded Ti-C changes, resulting in atomic displacements that could be related 
(i) Bond contracting between $\mathrm{Ti}$ and $\mathrm{C}$

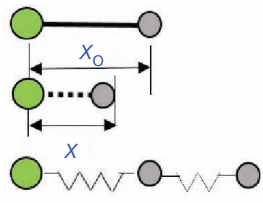

(ii) Bond contracting between $\mathrm{Ti}-\mathrm{C}$ and surface group termination

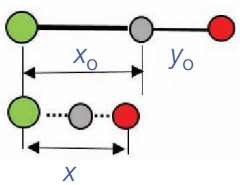

(a)

Before compression

Load

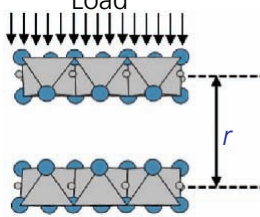

After compression

Load

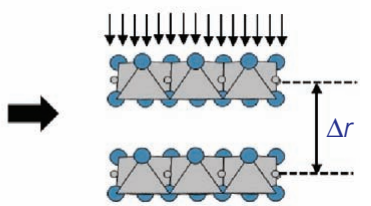

r, interlayer distance or distance between two atoms

(b)

Cobblestone model
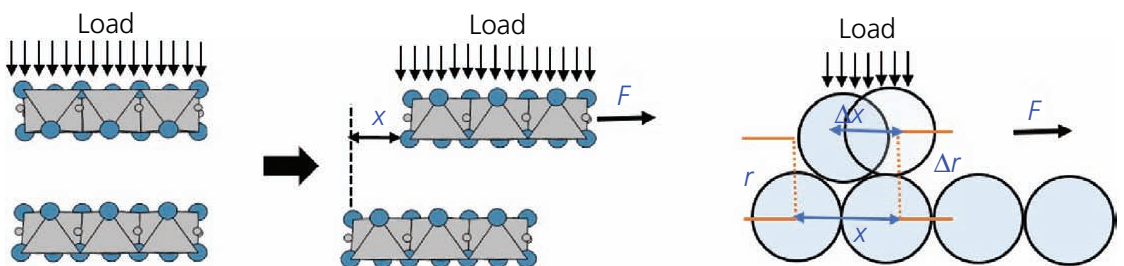

$F$, force; $x$, displacement due to shearing of the sheets

(c)

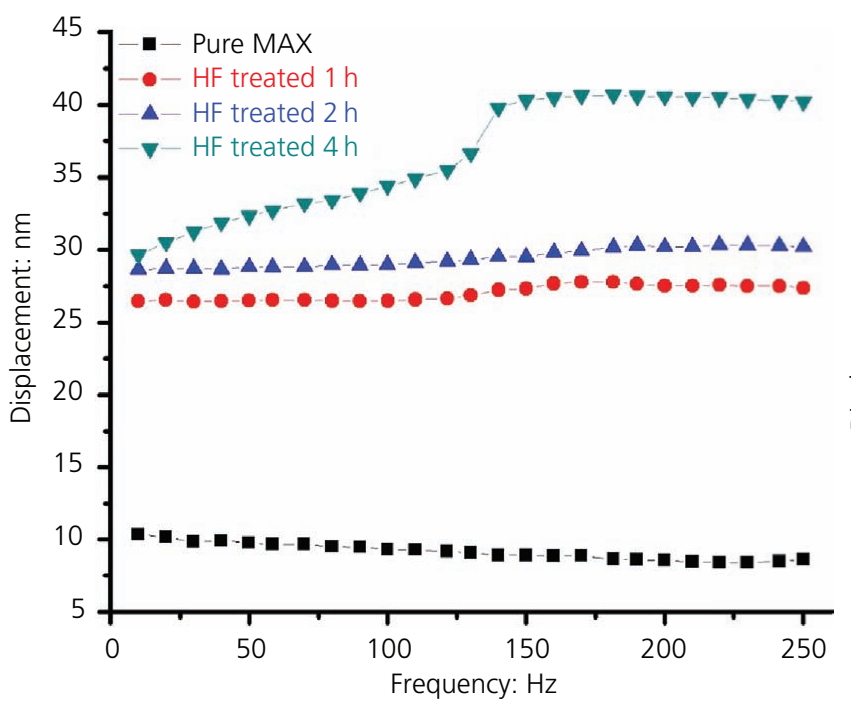

(d)

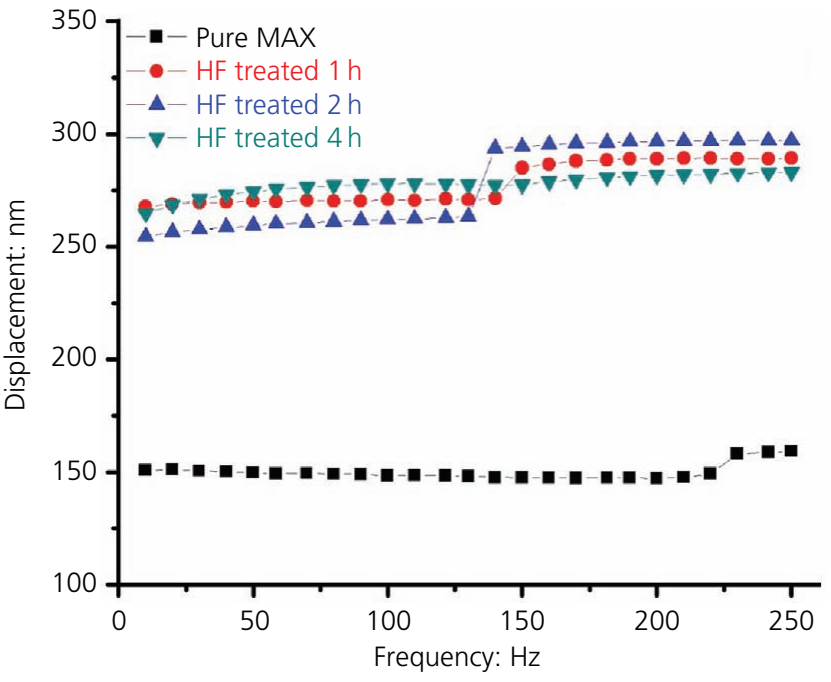

(e)

Figure 4. (a) Schematic diagram describing the intralayer deformation mechanism; further, the interlayer mechanism is described by (b) compression between the sheets and (c) shearing/sliding between the sheets. Change in displacement with varying frequency upon mechanical loadings of (d) 1 and (e) $10 \mu \mathrm{N}$. Energy change with varying frequency upon mechanical loading of (f) 1 and (g) $10 \mu \mathrm{N}$ (continued on next page) 


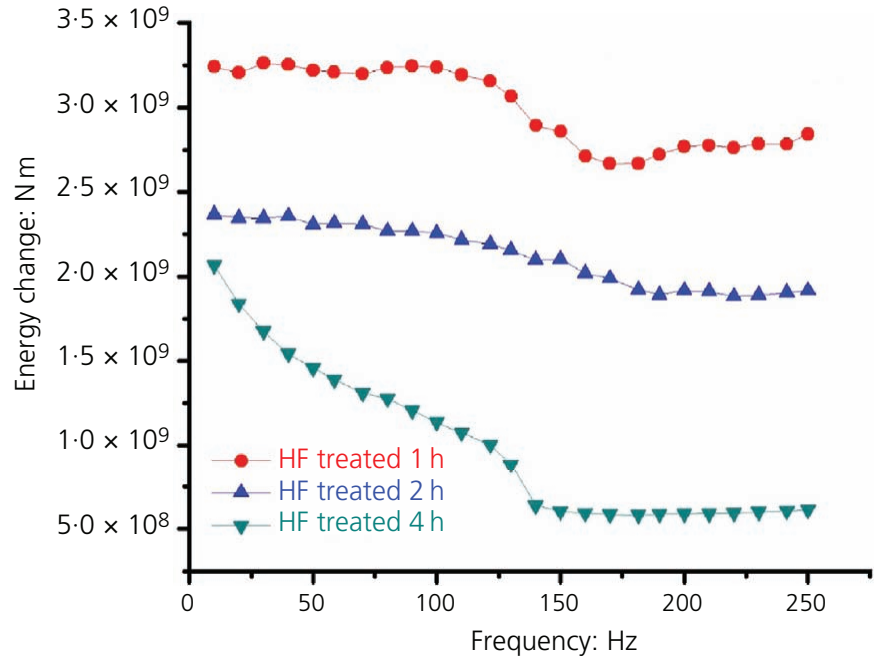

(f)

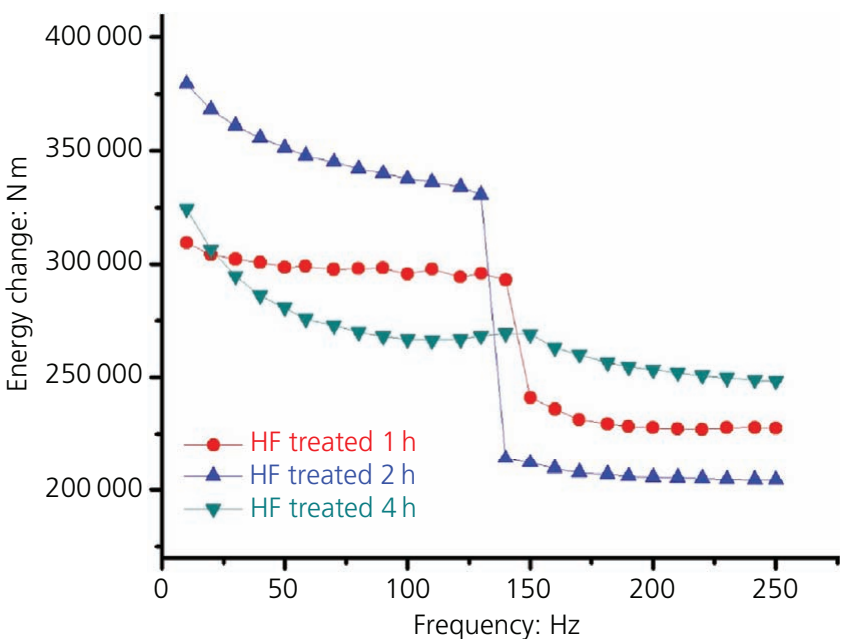

(g)

Figure 4. Continued

to the simple harmonic equation in molecular mechanics. ${ }^{49}$ Figure 4(a) illustrates the difference in the bond length of two covalently bonded atoms. The energy $\left(E_{\mathrm{I}}\right)$ required for stretching or contracting is shown by the equation

3. $E_{\mathrm{I}}=k_{\mathrm{s}}\left(x-x_{\mathrm{o}}\right)^{2}$

where $k_{\mathrm{s}}$ is the spring constant; $x_{\mathrm{o}}$ is the natural bond length between two atoms; and $x$ is the bond length when stretched. This equation can be further modified for the MXene sheet terminated with surface groups $(-\mathrm{OH},-\mathrm{F}$ or $-\mathrm{O})$ as follows

4. $E_{\mathrm{I}}=k_{\mathrm{s}}\left[x-\left(x_{\mathrm{o}}+y_{\mathrm{o}}\right)\right]^{2}$

where $k_{\mathrm{s}}$ is the spring constant; $x_{\mathrm{o}}$ is the natural bond length between two atoms; $y_{\mathrm{o}}$ is the natural bond length between an atom and a functional group; and $x$ is the bond length when stretched. The preceding relationships indicate that the energy dissipation along the in-plane direction in the MXene sheet when subjected to tensile/compressive loading is due to the bond stretching or contracting between $\mathrm{Ti}-\mathrm{C}$ and $\mathrm{Ti}-\mathrm{C}-\mathrm{OH}^{-} / \mathrm{F}^{-} / \mathrm{O}^{-}$.

Table 1. Calculated energy for bond contracting of pure and functionalized titanium carbide

\begin{tabular}{|cccc} 
Composition & $\begin{array}{c}\text { Bond } \\
\text { length }\left(\boldsymbol{x}_{\mathrm{o}}\right): \\
\times 10^{-10} \mathbf{m}\end{array}$ & $\begin{array}{c}\text { Spring } \\
\text { constant } \\
(\boldsymbol{k}): \mathbf{N} / \mathbf{m}\end{array}$ & $\begin{array}{c}\text { Energy } \\
\left(\boldsymbol{E}_{1}\right): \mathbf{N} \mathbf{~ m}\end{array}$ \\
\hline $\begin{array}{l}\text { MXene (titanium carbide) } \\
(\mathrm{Ti}-\mathrm{C})\end{array}$ & 2.09 & 125 & 0.3125 \\
$\mathrm{Ti}_{2} \mathrm{CO}_{2}(\mathrm{Ti}-\mathrm{C})$ & 2.17 & 227 & 0.5675 \\
$\mathrm{Ti}_{2} \mathrm{CO}_{2}(\mathrm{Ti}-\mathrm{O})$ & 1.96 & 227 & 0.0908 \\
\hline
\end{tabular}

This energy is quantified for pure and functionalized titanium carbide from the theoretical bond length and available $k_{\mathrm{s}}$ values from the literature. ${ }^{25,50}$ The bond length change values of -OH/-F-functionalized titanium carbide are not available in the literature. The change in bond length for pure and oxygenfunctionalized titanium carbide when subjected to different loadings is assumed to increase by $0 \cdot 05 / 0 \cdot 02 \AA .^{51}$ The calculated energy $\left(E_{\mathrm{I}}\right)$ values are tabulated in Table 1 , where the oxygenfunctionalized titanium carbide requires $80 \%$ higher energy for bond contracting than pure titanium carbide.

(b) Interlayer - compression between MXene sheets: titanium carbide MXene sheets are loosely held together by weak van der Waals forces, and energy dissipation along the interlayer direction could possibly be related to two mechanisms: (i) compression between the sheets and (ii) shearing/sliding between the sheets. First, compression of sheets due to weak van der Waals forces between titanium carbide sheets is discussed, as shown in Figure 4(b). This mechanism was confirmed by performing cyclic compressive loading-unloading-reloading of MXene (shown in Video V1 in the online supplementary material). In the weak van der Waals forces, the attractive force between two atoms is referred to as the London dispersion force. ${ }^{38}$ From the interaction energy $\left(E_{\mathrm{II}}\right)$ of van der Waals forces of two atoms, the weak interaction force between the atoms was derived and modified by Hamaker as given in the following

5. $F=\frac{A R}{12 r^{2}}$

where $R$ is the radius of the spheres; $r$ is the distance between two atoms; and $A$ is referred to as the Hamaker constant, ranging from $10^{-11}$ to $10^{-13}$ ergs. $^{38}$ The preceding expression was modified by 
considering the weak interaction between two flat surfaces, and the interaction force $(F)$ was given by

$$
\text { 6. } F=\frac{A}{6 \pi r^{3}}
$$

Thus, the distance between two flat surfaces, ' $r$ ', could be directly correlated with the interlayer distance between the MXene sheets. By differentiating Equation 6, the energy change can be described as follows

7. $E_{\mathrm{II}}=\frac{A}{2 \pi r^{4}}$

where the energy change is proportional to $1 / r^{4}$. This equation suggests that the energy change increases with decreasing interlayer distance $(r)$. Enhanced energy dissipation results in a stronger damping effect due to the presence of weak van der Waals forces between the MXene sheets. This energy change was quantified by using the displacement data (Figures 4(d) and 4(e)) measured during nano-DMA for both loads $(1$ and $10 \mu \mathrm{N})$. Using the theoretical monolayer thickness of titanium carbide and displacement data, the number of titanium carbide monolayers that interacted during damping was calculated, as shown in Table 2, and each monolayer was separated by weak van der Waals forces (an interlayer distance of titanium carbide - included in Table $\mathrm{S} 1$ in the online supplementary material). ${ }^{50}$ The displacement data were substituted into Equation 7, and the corresponding energy changes are shown in Figures 4(f) and 4(g). The MAX phase was not considered for this energy calculation, as this equation was derived based on the assumptions that the sheets were loosely held together by weak van der Waals forces. The energy change during damping $(1 \mu \mathrm{N}$ load) for the $1 \mathrm{~h}$ hydrofluoric acid-treated sample was 1.25 and 1.5 times higher than those for the 2 and $4 \mathrm{~h}$ hydrofluoric acid-treated samples, respectively. This observation is in agreement with $\tan \delta$ values of hydrofluoric acid-treated samples, where the $1 \mathrm{~h}$ MXene has the highest $\tan \delta$ of $0 \cdot 35$. However, the $10 \mu \mathrm{N}$ load energy change for the $1 \mathrm{~h}$ hydrofluoric acid-treated sample decreases beyond a frequency of $150 \mathrm{~Hz}$ and a displacement of $270 \mathrm{~nm}$. Thus, the energy change for $1 \mathrm{~h}$ hydrofluoric acid-treated sample of $10 \mu \mathrm{N}$ load is not in agreement with the $\tan \delta$ value, indicating that compression of MXene sheets will not be a dominating mechanism after a specific displacement and high load. With higher load, the dominating mechanism will be interlayer shearing/sliding between the sheets.

(c) Interlayer - sliding/shearing between the sheets: the second interlayer mechanism due to weak van der Waals forces is the shearing/sliding of MXene sheets. This mechanism is explained based on the cobblestone model (Figure 4(c)).$^{52}$ It states that two surfaces slide against each other when subjected to an external load. During sliding, because of the weak van der Waals forces between the surfaces, the layers are separated or attracted to each other. The energy is dissipated in this process of surface separation or attraction. This energy is described as follows

8. $\operatorname{energy}\left(E_{\mathrm{III}}\right)=F \Delta x \rightarrow F \Delta x=4 \Delta \gamma A \frac{\Delta r}{r}$

where $\gamma$ is the surface energy; $A$ is the contact area; $r$ is the initial surface separation; $\Delta r$ is the small surface separation after sliding; and $\Delta \gamma=\gamma_{\text {separation }}-\gamma_{\text {attraction. }}$. From the preceding equation, the shear strength $\left(\mathrm{S}_{\mathrm{c}}\right)$ at the interface can be evaluated by

9. $S_{\mathrm{c}}=\frac{F}{A} \rightarrow S_{\mathrm{c}}=\frac{4 \Delta \gamma \Delta r}{\Delta x r} \rightarrow S_{\mathrm{c}}=\frac{4 \gamma \varepsilon \Delta r}{\Delta x r}$

where the coefficient of dissipation $\varepsilon=\Delta \gamma / \gamma$. This $r$ could be related to the distance between two atoms, which in turn describes the interlayer distance. Also, previous studies have shown that with hydrofluoric acid treatment, the surface energy increases compared to pure MAX, and with surface functionalization of $-\mathrm{O}$, the surface energy increases compared to that of $-\mathrm{OH} /-\mathrm{F}^{23,50}$ The coefficient of dissipation $\varepsilon$ delineates the fraction of energy loss as the interlayer distance between the MXene sheets increases or decreases. Further, the interlayer distance is inversely proportional to shear strength, indicating that the sliding of layers occurs more frequently as the interlayer distance decreases. Thus, during damping, the energy could also be dissipated by sliding of MXene sheets. The sliding of MXene sheets was evaluated by conducting sliding tribological tests on MAX and MXene (see details in the online supplementary material). The COF value was $0 \cdot 18$ for the pure MAX phase powder at $1 \mathrm{~N}$ load. The COF significantly decreased to $0 \cdot 019$ for $1 \mathrm{~h}$ hydrofluoric acid-treated powder $(1 \mathrm{~N})$; with 2 and $4 \mathrm{~h}$ of hydrofluoric acid treatment $(1 \mathrm{~N})$,

Table 2. Total number of layers interacting during damping, calculated from the monolayer thickness and average displacement measured during nano-DMA for hydrofluoric acid-treated samples

\begin{tabular}{|c|c|c|c|c|c|}
\hline Load: $\mu \mathrm{N}$ & $\begin{array}{l}\text { Hydrofluoric acid } \\
\text { treatment duration: } \mathbf{h}\end{array}$ & $\begin{array}{l}\text { Theoretical thickness of the } \\
\text { monolayer (titanium carbide): } \mathrm{nm}\end{array}$ & $\begin{array}{c}\text { Average } \\
\text { displacement: } \mathrm{nm}\end{array}$ & $\begin{array}{l}\text { Number of monolayers } \\
\text { interacting during damping }\end{array}$ & $\tan \delta$ \\
\hline \multirow[t]{3}{*}{1} & 1 & 0.231 & 27 & 54 & $0.10-0.35$ \\
\hline & 2 & 0.231 & 30 & 60 & $0.01-0.28$ \\
\hline & 4 & 0.231 & 37 & 74 & $0.04-0.17$ \\
\hline \multirow[t]{3}{*}{10} & 1 & 0.231 & 278 & 556 & $0.06-0.38$ \\
\hline & 2 & 0.231 & 277 & 554 & $0.06-0.28$ \\
\hline & 4 & 0.231 & 278 & 556 & $0.04-0.15$ \\
\hline
\end{tabular}


the COF increased to $0 \cdot 021$ and $0 \cdot 079$, respectively. It should be noted that the COF for MXene was $56-89 \%(0 \cdot 079-0 \cdot 019)$ lower than that for MAX $(0 \cdot 18)$, which is ascribed to the interlayer shearing/sliding of MXene sheets due to the weak van der Waals forces between the layers. Further, $1 \mathrm{~h}$ hydrofluoric acid-treated MXene showed superior lubrication properties compared to 2 and $4 \mathrm{~h}$ hydrofluoric acid-treated MXene, which is due to the increase in interlayer spacing with extended hydrofluoric acid treatment. The COF data concur with damping $\tan \delta$ values, with $1 \mathrm{~h}$ treated MXene demonstrating an excellent damping behavior. Recently, a similar behavior of shear-induced slipping of $\mathrm{Ti}_{3} \mathrm{C}_{2}$ MXene nanosheets under pressure was observed, which further substantiates the shearing of titanium carbide sheets in the present work. ${ }^{27}$ Therefore, the interlayer mechanism of shearing/sliding of MXene sheets for energy dissipation is supported by the tribological study.

With increasing hydrofluoric acid treatment duration, the array of MXene sheets in the stack decreases, which explains the weak van der Waals forces gradually waning between the sheets. Thus, the intralayer and interlayer mechanisms become less active with increasing hydrofluoric acid treatment duration. In addition, the defects formed during hydrofluoric acid treatment will influence the damping response. The defect concentration will increase with hydrofluoric acid treatment duration. ${ }^{53}$ From the displacement data measured during nano-DMA (Figure 4(d)), at a low load of $1 \mu \mathrm{N}$, the displacement of MXene varied between 26 and $42 \mathrm{~nm}$, and, based on the calculations from Table 1, some MXene sheets interact during damping are well below the thickness of separated layers $(70-150 \mathrm{~nm})$. Since the displacement was within the MXene layer, intralayer bond contracting and interlayer compression would be the dominant energy-dissipation mechanisms at a lower load of $1 \mu \mathrm{N}$. With a dynamic load of $10 \mu \mathrm{N}$, the displacement was above $250 \mathrm{~nm}$ for all the hydrofluoric acid-treated samples. This indicates that the displacement would be higher than $250 \mathrm{~nm}$ at the higher loads of 1 and $5 \mathrm{mN}$. With the increase in applied force, the displacement proceeds through the interlayer gap between the MXene sheets; at this stage, the energy dissipation occurs through the interlayer compression/sliding between the sheets. Thus, at larger loads, the dominant energy dissipation will be a combination of all three mechanisms: (a) intralayer - bond contracting; $(b)$ interlayer compression between MXene sheets; and $(c)$ interlayer - sliding/ shearing between the sheets. Therefore, the combination of both intralayer and interlayer mechanisms in MXene will surpass the damping behavior of the pure MAX phase and MXene will emerge as an excellent damping material.

\section{Conclusion}

In conclusion, this first experimental study on damping reveals the superior (200\%) and stable (50 000 cycles) damping behavior of hydrofluoric acid-treated MXene compared to pure MAX phase. The excellent damping characteristics of MXene are ascribed to intralayer bond contracting of Ti-C/Ti-C-OH $/ \mathrm{F}^{-} / \mathrm{O}^{-}$ and interlayer compression/shearing due to weak van der Waals forces. These experimental findings on the mechanical damping property of MXene can lead to several engineering applications from nanomechanical devices to reinforcement in antivibration composites.

\section{Acknowledgement}

The authors thank the Advanced Materials Engineering Research Institute at Florida International University for the characterization facilities.

\section{REFERENCES}

1. Geim AK and Novoselov KS (2007) The rise of graphene. Nature Materials 6(3): 183-191.

2. Hamm M and Hess O (2013) Two two-dimensional materials are better than one. Science 340(6138): 1298-1299.

3. Bhimanapati GR, Lin Z, Meunier V et al. (2015) Recent advances in two-dimensional materials beyond graphene. ACS Nano 9(12): 11509-11539.

4. Xu M, Liang T, Shi M and Chen H (2013) Graphene-like two-dimensional materials. Chemical Reviews 113(5): 3766-3798.

5. Lee C, Wei X, Kysar JW and Hone J (2008) Measurement of the elastic properties and intrinsic strength of monolayer graphene. Science 321(5887): 385-388.

6. Wang L, Meric I, Huang PY et al. (2013) One-dimensional electrical contact to a two-dimensional material. Science 342(6158): 614-617.

7. Pomerantseva E and Gogotsi $Y$ (2017) Two-dimensional heterostructures for energy storage. Nature Energy 2(7): article 17089.

8. Liang J, Wang Y, Huang $Y$ et al. (2009) Electromagnetic interference shielding of graphene/epoxy composites. Carbon 47(3): 922-925.

9. Deng DH, Novoselov KS, Fu Q et al. (2016) Catalysis with twodimensional materials and their heterostructures. Nature Nanotechnology 11(3): 218-230.

10. Naguib M, Kurtoglu M, Presser V et al. (2011) Two-dimensional nanocrystals produced by exfoliation of $\mathrm{Ti}_{3} \mathrm{AlC}_{2}$. Advanced Materials 23(37): 4248-4253.

11. Naguib M, Mochalin VN, Barsoum MW and Gogotsi $Y$ (2014) 25th anniversary article: MXenes: a new family of two-dimensional materials. Advanced Materials 26(7): 992-1005.

12. Barsoum MW (2000) The $\mathrm{M}_{N+1} \mathrm{AX}_{N}$ phases: a new class of solids: thermodynamically stable nanolaminates. Progress in Solid State Chemistry 28(1-4): 201-281.

13. Barsoum MW (2006) Physical properties of MAX phases. In Encyclopedia of Materials Science and Technology (Buschow KHJ, Cahn RW, Flemings MC et al. (eds)). Elsevier, Amsterdam, the Netherlands, pp. 1-11.

14. Naguib M, Mashtalir O, Carle J et al. (2012) Two-dimensional transition metal carbides. ACS Nano 6(2): 1322-1331.

15. Naguib M, Come J, Dyatkin B et al. (2012) MXene: a promising transition metal carbide anode for lithium-ion batteries. Electrochemistry Communications 16(1): 61-64.

16. Peng YY, Akuzum B, Kurra N et al. (2016) All-MXene (2D titanium carbide) solid-state microsupercapacitors for on-chip energy storage. Energy \& Environmental Science 9(9): 2847-2854.

17. Peng Q, Guo J, Zhang Q et al. (2014) Unique lead adsorption behavior of activated hydroxyl group in two-dimensional titanium carbide. Journal of the American Chemical Society 136(11): 4113-4116.

18. Wang F, Yang C, Duan C et al. (2013) An organ-like titanium carbide material (MXene) with multilayer structure encapsulating hemoglobin for a mediator-free biosensor. Journal of the Electrochemical Society 162(1): B16-B21.

19. Shahzad F, Alhabeb M, Hatter CB et al. (2016) Electromagnetic interference shielding with 2D transition metal carbides (MXenes). Science 353(6304): 1137-1140. 
20. Hu Q, Sun D, Wu Q et al. (2013) MXene: a new family of promising hydrogen storage medium. Journal of Physical Chemistry A 117(51): $14253-14260$.

21. Jhon YI, Koo J, Anasori B et al. (2017) Metallic MXene saturable absorber for femtosecond mode-locked lasers. Advanced Materials 29(40): 1702496

22. Kurtoglu M, Naguib M, Gogotsi Y and Barsoum MW (2012) First principles study of two-dimensional early transition metal carbides. MRS Communications 2(4): 133-137.

23. Wang S, Li JX, Du YI and Cui C (2014) First-principles study on structural, electronic and elastic properties of graphene-like hexagonal $\mathrm{Ti}_{2} \mathrm{C}$ monolayer. Computational Materials Science 83: 290-293.

24. Guo Z, Zhou J, Si C and Sun Z (2015) Flexible two-dimensional $\mathrm{Ti}_{n+1} \mathrm{C}_{n}(n=1,2$ and 3$)$ and their functionalized MXenes predicted density functional theories. Physical Chemistry Chemical Physics 17(23): 15348-15354.

25. Yorulmaz U, Ozden A, Perkgoz NK, Ay F and Sevik C (2016) Vibrational and mechanical properties of single layer MXene structures: a first-principles investigation. Nanotechnology 27(33): article 335702.

26. Lipatov A, Lu H, Alhabeb M et al. (2018) Elastic properties of 2D $\mathrm{Ti}_{3} \mathrm{C}_{2} \mathrm{~T}_{x}$ MXene monolayers and bilayers. Science Advances 4(6): article eaat0491.

27. Ghidiu M, Kota S, Drozd V and Barsoum MW (2018) Pressureinduced shear and interlayer expansion in $\mathrm{Ti}_{3} \mathrm{C}_{2}$ MXene in the presence of water. Science Advances 4(1): article eaao6850.

28. Ling Z, Ren CE, Zhao MQ et al. (2014) Flexible and conductive MXene films and nanocomposites with high capacitance. Proceedings of the National Academy of Sciences of the United States of America 111(47): 16676-16681.

29. Naguib M, Saito T, Lai S et al. (2016) $\mathrm{Ti}_{3} \mathrm{C}_{2} \mathrm{~T}_{x}$ (MXene)-polyacrylamide nanocomposite films. RSC Advances 6(76): 72069-72073.

30. Zhang H, Fu ZH, Legut D, Germann TC and Zhang RF (2017) Stacking stability and sliding mechanism in weakly bonded 2D transition metal carbides by van der Waals force. RSC Advances 7(88): 55912-55919.

31. Zhang $D$, Ashton $M$, Ostadhossein A et al. (2017) Computational study of low interlayer friction in $\mathrm{Ti}_{n+1} \mathrm{C}_{n}(n=1,2$, and 3) MXene. ACS Applied Materials \& Interfaces 9(39): 34467-34479.

32. Liu $Y$, Zhang $X$, Dong $S, Y e Z$ and Wei $Y$ (2017) Synthesis and tribological property of $\mathrm{Ti}_{3} \mathrm{C}_{2} \mathrm{~T}_{X}$ nanosheets. Journal of Materials Science 52(4): 2200-2209.

33. Yang J, Chen BB, Song HJ, Tang H and Li CS (2014) Synthesis, characterization, and tribological properties of two-dimensional $\mathrm{Ti}_{3} \mathrm{C}_{2}$. Crystal Research and Technology 49(11): 926-932.

34. Zhang $\mathrm{XH}$, Xue MQ, Yang $\mathrm{XH}$ et al. (2015) Preparation and tribological properties of $\mathrm{Ti}_{3} \mathrm{C}_{2}(\mathrm{OH})_{2}$ nanosheets as additives in base oil. RSC Advances 5(4): 2762-2767.

35. Zhang H, Wang L, Chen Q et al. (2016) Preparation, mechanical and anti-friction performance of MXene/polymer composites. Materials \& Design 92: 682-689.

36. Zhang X, Guo Y, Li Y, Liu Y and Dong S (2019) Preparation and tribological properties of potassium titanate- $\mathrm{Ti}_{3} \mathrm{C}_{2} \mathrm{~T}_{x}$ nanocomposites as additives in base oil. Chinese Chemical Letters 30(2): 502-504

37. Mai YJ, Li YG, Li SL et al. (2019) Self-lubricating $\mathrm{Ti}_{3} \mathrm{C}_{2}$ nanosheets/ copper composite coatings. Journal of Alloys and Compounds 770: 1-5.

38. Lahiri D, Das S, Choi W and Agarwal A (2012) Unfolding the damping behavior of multilayer graphene membrane in the low-frequency regime. ACS Nano 6(5): 3992-4000.

39. Nautiyal P, Boesl B and Agarwal A (2018) The mechanics of energy dissipation in a three-dimensional graphene foam with macroporous architecture. Carbon 132: 59-64.

40. Li JX, Du YL, Huo CX, Wang S and Cui C (2015) Thermal stability of two-dimensional $\mathrm{Ti}_{2} \mathrm{C}$ nanosheets. Ceramics International 41(2): 2631-2635.

41. Nautiyal P, Embrey L, Boesl B and Agarwal A (2017) Multi-scale mechanics and electrical transport in a free-standing $3 \mathrm{D}$ architecture of graphene and carbon nanotubes fabricated by pressure assisted welding. Carbon 122: 298-306.

42. Agrawal R, Nieto A, Chen H, Mora M and Agarwal A (2013) Nanoscale damping characteristics of boron nitride nanotubes and carbon nanotubes reinforced polymer composites. ACS Applied Materials \& Interfaces 5(22): 12052-12057.

43. Radovic M, Ganguly A, Barsoum MW et al. (2006) On the elastic properties and mechanical damping of $\mathrm{Ti}_{3} \mathrm{SiC}_{2}, \mathrm{Ti}_{3} \mathrm{GeC}_{2}$, $\mathrm{Ti}_{3} \mathrm{Si}_{0.5} \mathrm{Al}_{0.5} \mathrm{C}_{2}$ and $\mathrm{Ti}_{2} \mathrm{AlC}$ in the $300-1573 \mathrm{~K}$ temperature range. Acta Materialia 54(10): 2757-2767.

44. Zhou AG, Barsoum MW, Basu S, Kalindini SR and El-Raghy T (2006) Incipient and regular kink bands in fully dense and $10 \mathrm{vol} . \%$ porous $\mathrm{Ti}_{2} \mathrm{AlC}$. Acta Materialia 54(6): 1631-1639.

45. Amini S, Ni C and Barsoum MW (2009) Processing, microstructural characterization and mechanical properties of a $\mathrm{Ti}_{2} \mathrm{AlC} /$ nanocrystalline $\mathrm{Mg}$-matrix composite. Composites Science and Technology 69(3-4): 414-420.

46. Gruber J, Lang AC, Griggs J et al. (2016) Evidence for bulk ripplocations in layered solids. Scientific Reports 6: article 33451.

47. Barsoum MW and Tucker GJ (2017) Deformation of layered solids: ripplocations not basal dislocations. Scripta Materialia 139: 166-172.

48. Nautiyal P, Boesl B and Agarwal A (2017) Harnessing three dimensional anatomy of graphene foam to induce superior damping in hierarchical polyimide nanostructures. Small 13(10): 1603473.

49. Fyta M (2016) Computational Approaches in Physics. Morgan \& Claypool, San Rafael, CA, USA.

50. Bai Y, Zhou K, Srikanth N et al. (2016) Dependence of elastic and optical properties on surface terminated groups in two-dimensional MXene monolayers: a first principles study. RSC Advances 6(42): 35731-35739.

51. Khan SA, Amin B, Gan LY and Ahmad I (2017) Strain engineering of electronic structures and photocatalytic responses of MXenes functionalized by oxygen. Physical Chemistry Chemical Physics 19(22): 14738-14744.

52. Hu Y, Ma TB and Wang H (2013) Energy dissipation in atomic-scale friction. Friction 1(1): $24-40$.

53. Sang $X, X i e ~ Y$, Lin MW et al. (2016) Atomic defects in monolayer titanium carbide $\left(\mathrm{Ti}_{3} \mathrm{C}_{2} \mathrm{~T}_{x}\right)$ MXene. ACS Nano 10(10): 9193-9200.

\section{How can you contribute?}

To discuss this paper, please submit up to 500 words to the journal office at journals@ice.org.uk. Your contribution will be forwarded to the author(s) for a reply and, if considered appropriate by the editor-in-chief, it will be published as a discussion in a future issue of the journal.

ICE Science journals rely entirely on contributions from the field of materials science and engineering. Information about how to submit your paper online is available at www.icevirtuallibrary.com/page/authors, where you will also find detailed author guidelines. 